\title{
Editorial
}

\section{School Bullying and Victimization}

\author{
Byongook Moon, ${ }^{1}$ John McCluskey, ${ }^{2}$ and Christopher Schreck ${ }^{2}$ \\ ${ }^{1}$ Department of Criminal Justice, University of Texas, San Antonio, TX 78207, USA \\ ${ }^{2}$ Department of Criminal Justice, Rochester Institute of Technology, Rochester, NY 14623, USA
}

Correspondence should be addressed to Byongook Moon; byongook.moon@utsa.edu

Received 28 July 2013; Accepted 28 July 2013

Copyright (c) 2013 Byongook Moon et al. This is an open access article distributed under the Creative Commons Attribution License, which permits unrestricted use, distribution, and reproduction in any medium, provided the original work is properly cited.

School bullying and victimization is a serious social and school issue. Negative consequences for victims, bullies, and overall school climate have been documented across different contexts, cultures, and countries. The extant research indicates that bullying is a significant predictor of delinquency and criminal behaviors. Research also indicates that bullying victims are more likely to suffer from depression, anxiety, and learning difficulties. Together, the findings suggest avenues for theoretical testing and intervention programs to reduce bullying and ameliorate its consequences. This special issue seeks to enhance our understanding and knowledge of these issues, focusing on the etiology of bullying and victimization, and to suggest specific policy implications for addressing bullying and victimization. We present nine articles that examine some of these issues, using samples of diverse populations from Cyprus, England, Greece, and the United States.

Dr. A. HA. Peguero and colleagues, using a sample of American adolescents, examined the relationship between routine activities (academics, sports, clubs, and misbehavior) and school victimization across distinct school contexts and concluded that some of these routine activities (i.e., engagement in school-based misbehavior) are significantly related to school victimization across urban, rural, and suburban school contexts. The study also indicates the significant effect of adolescents' race on school victimization and suggests the necessity of developing violence intervention and prevention programs specifically geared toward racial and ethnic minority students.

The study by Dr. S. Jeong et al. tested whether school individual characteristics and environmental factors, including preventive measures, are related to bullying victimization in a sample of youth from the United States. The study found that students are more likely to be victimized when they report gangs/guns at school but less likely to be victimized if they perceive greater fairness of school ruler, or when security officers are present in school hallways. With respect to individual characteristics, those involved in fighting were more likely to report physical, emotional, and combined incidents of bullying relative to respondents not involved in fighting. The results suggest the importance of creating and maintaining safe school environment as it documents the role of security and perceived fairness in preventing school bullying and victimization and also recognizes the importance of individual characteristics and behaviors as predictors of victimization.

M. Vlachou and colleagues, using a sample of 167 children in Greece, explored the prevalence of school bullying among preschool children in Greece by employing a multimethod approach, especially using peer nominations, selfand teacher reports, and natural observation. They found that the frequencies of bullying vary greatly depending on how bullying is measured and indicate the importance of designing and employing reliable and valid methodological approaches to better understand the prevalence of bullying, especially among preschool population.

With a sample of 325 youths in England, the research conducted by Dr. T. Völlink and colleagues examined the role of coping strategies in the relationship between cyberbullying and depressive feelings/health complaints. Interestingly, they found that youths using a problem-focused coping are less likely to report health complaints and depressive feelings, 
while those employing emotion-focused coping are more likely to experience negative health outcomes when they are bullied online, contrary to previous findings on traditional bullying. This finding raises a question over the effectiveness of emotion-focused coping strategy for victims of cyberbullying.

Dr. S. L. Johnson and colleagues tested whether bullying victims' bystander perceptions and school climate are related to their responses to bullying in a sample of 6,493 adolescents in the United States. The research indicates that bullying victims are less likely to choose aggressive responses to bullying and more likely to seek support from school staff when they report higher levels of school safety and connectedness. The findings suggest the importance of developing bullying prevention programs, focusing on school norms, climate, and cultures.

Dr. S. Jeong and B. H. Lee, using a sample of youth in the United States, investigated the relationship between individual/school level characteristics and peer victimization and found that gender, age, parental support, and school pressure are significantly related to victimization in the expected direction. Interestingly, the finding shows that students attending schools with bullying prevention programs are more likely to experience peer victimization, indicating the necessity of further research on the effectiveness of various bullying prevention programs.

The study conducted by Dr. P. R. Smokowski and colleagues examined the prevalence of school bullying and victimization and related risk factors among a sample of rural youth in the United States. The results indicate that the prevalence of bullying, especially extreme victimization, in rural schools is much higher than that found in many samples of urban and suburban youth. This finding reveals the severity of school bullying and victimization in rural schools and underscores the importance of understanding bullying dynamics within that context. Multivariate analyses indicate that individual factors are more strongly related to bullying victimization than school environmental factors. This is consistent with previous studies.

With a sample of 895 children in the United States, Drs. K. A. Fanti and S. N. Georgiou examined the differences among children involved in bullying in terms of parental relationship and academic performance and the transactional association between child-parental relationship and child behavioral problems. The results indicate that children identified as bully victim and occasional bully or victim are more likely to experience conflict with parents and poor academic performance, compared to uninvolved, bully only, and victim only groups. The transactional findings suggest a bidirectional association between bullying, victimization, and parent-child relationship, indicating dynamic and reciprocal interrelationship between child and parents over time.

The research conducted by Dr. M. Nikiforou and colleagues, using a sample of approximately 300 youth in Cyprus, investigated the relationship between parental/peer attachment and bullying and victimization. Consistent with the finding by Drs. K. A. Fanti and S. N. Georgiou, they found that adolescents with poor quality of attachment with parents and peers are more likely to engage in bullying as perpetrators or victims and its relationship is stronger for girls. They suggest the importance of developing bullying prevention programs geared toward improving quality of attachment with parents and peers.

These nine articles provide a substantial contribution to the growing literature on bullying and its causes, consequences, and prevention. They clearly illustrate the ubiquity of the problem across countries and rural, suburban, and urban locations. Overall, we believe that this special issue will contribute our understanding of school bullying and victimization and highlights the importance of developing comprehensive approaches to bullying prevention programs, rooted in a foundation of theory and research, as multiple factors at the individual and contextual level are found to be related to bullying and victimization.

Byongook Moon John McCluskey Christopher Schreck 

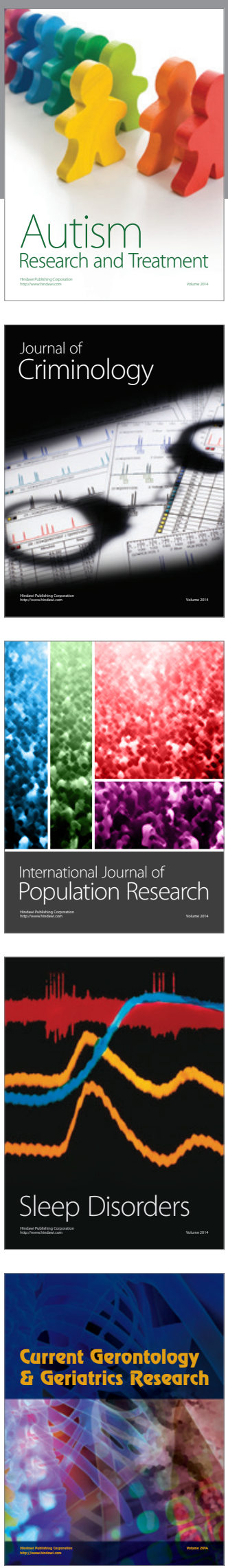
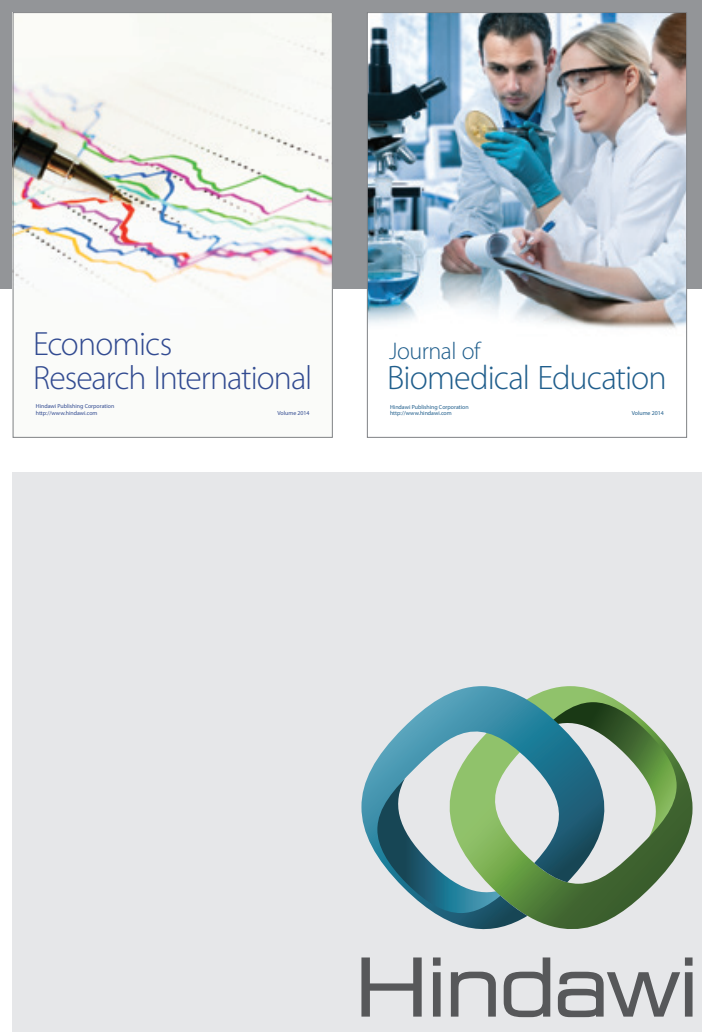

Submit your manuscripts at

http://www.hindawi.com
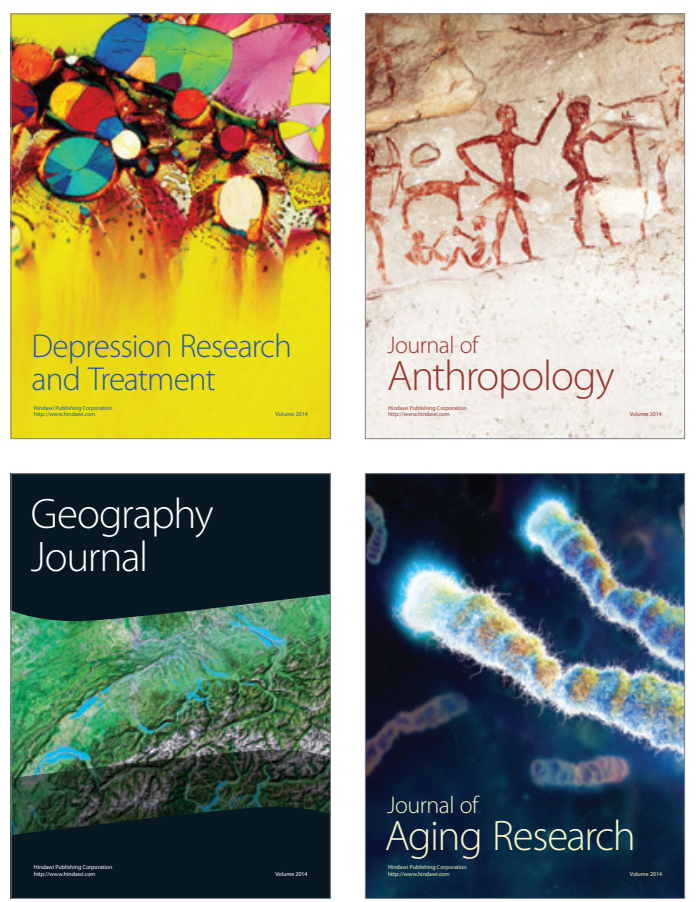
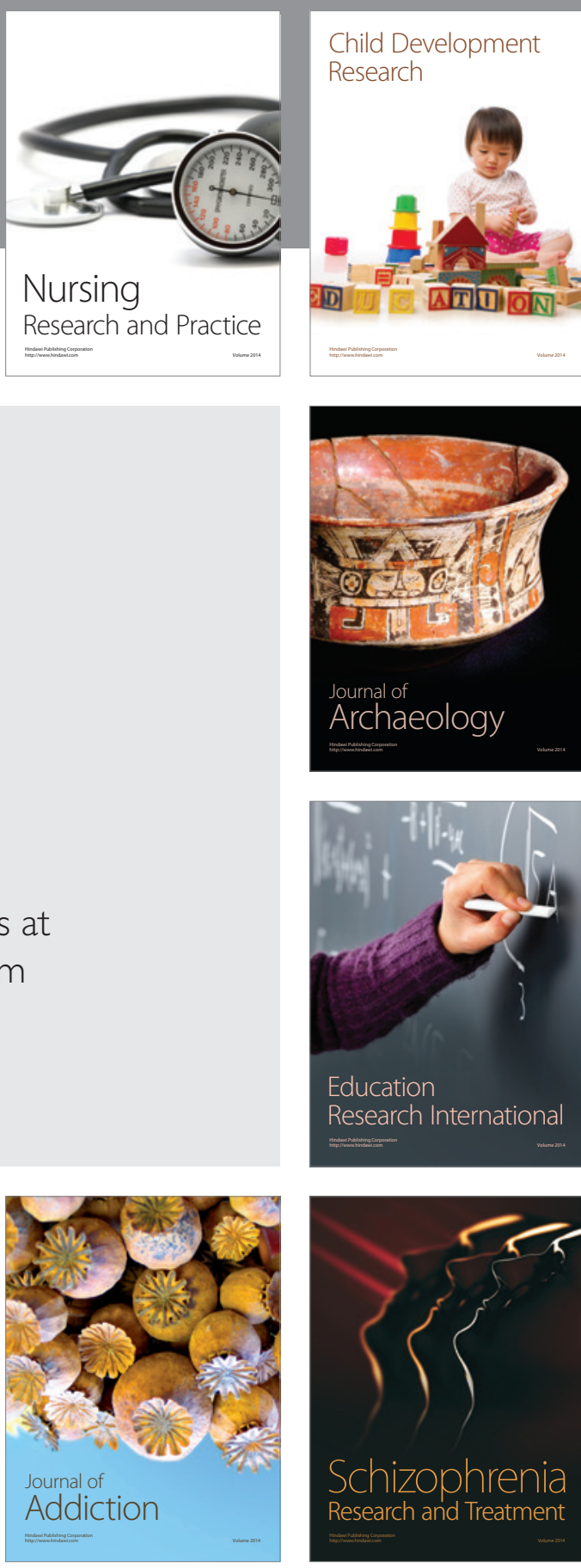

(D)
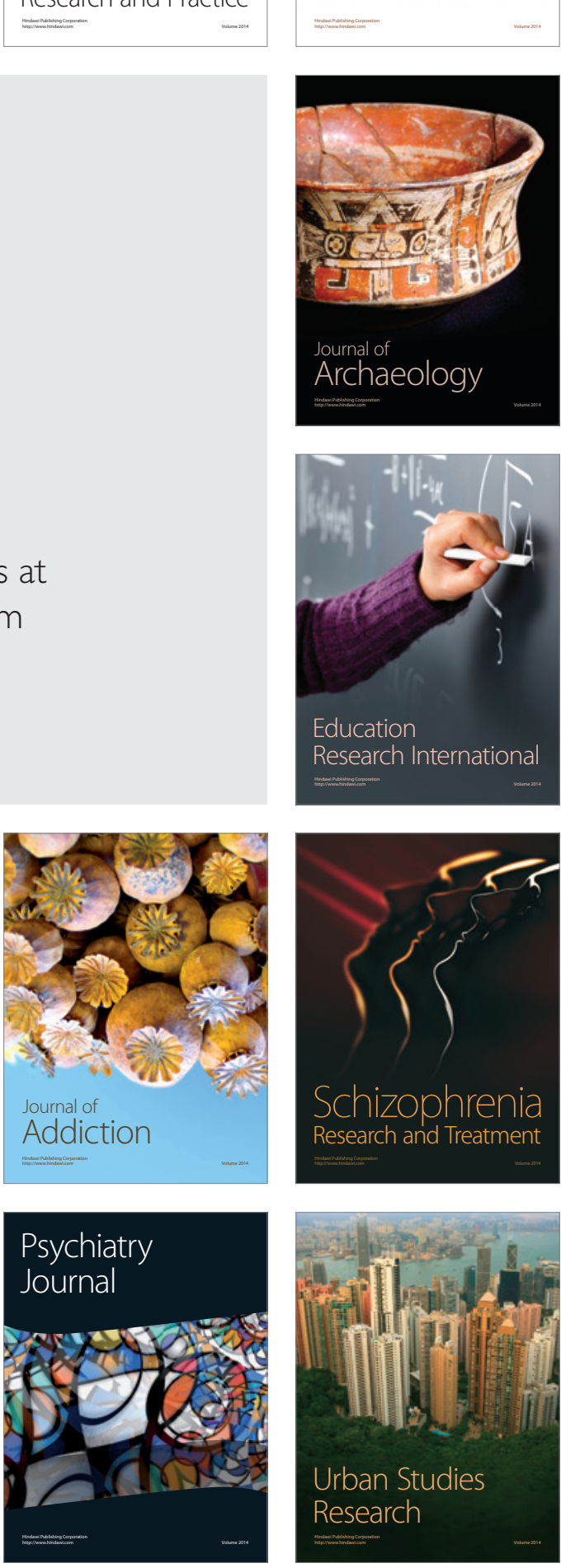